\title{
A thermo-elastic-plastic phase-field model for simulating the evolution and transition of adiabatic shear band. Part I. Theory and model calibration
}

\author{
T. Wang ${ }^{\mathrm{a}}$, Z.L. Liu' ${ }^{\mathrm{a}, *}$, Y.N. Cui ${ }^{\mathrm{a}}$, X. Ye ${ }^{\mathrm{b}}$, X.M. Liu' ${ }^{\mathrm{b}}$, R. Tian ${ }^{\mathrm{c}}$, Z. Zhuang ${ }^{\mathrm{a}, *}$ \\ a Applied Mechanics Lab., School of Aerospace Engineering, Tsinghua University, Beijing 100084, China \\ ${ }^{\mathrm{b}}$ LNM, Institute of Mechanics, Chinese Academy of Sciences, Beijing 100190, China \\ c Software Center for High Performance Numerical Simulation, Institute of Applied Physics and Computational Mathematics, Beijing 100088, China
}

\section{A R T I C L E I N F O}

\section{Keywords:}

Adiabatic shear band

Phase-field model

Fracture

Parameter calibration

Multi-field coupling

\begin{abstract}
A B S T R A C T
Adiabatic shear band (ASB) is an important failure mode of solid materials, especially for metal materials under high strain rate loading. In this study, a thermo-elastic-plastic phase-field model, which considers both damage softening and thermal softening, is established to simulate the formation of multiple ASBs and the transition from ASB to the fracture. How to select and calibrate the material parameters in the phase-field model is seldom clearly discussed in the current phase-field model when dealing with ASB. In this paper, the damage parameters in the phasefield method are calibrated using data from pure shear specimens, taking into account both the overall response of the structure and the local response in the ASB. As an application, the calibrated model is used to numerically study the evolution of the ASB of the hat specimen and the process of its transition to the fracture. The simulation results successfully explain the typical phenomena such as transient "hot spots" and double softening in the experiment. The numerical model we developed provides a reliable quantitative description for the evolution and width calculation of ASBs and lays a foundation for the further study of ASB.
\end{abstract}

\section{Introduction}

In common metal engineering materials, shear localization is one of the most important failure modes, which leads to the generation of the adiabatic shear band (ASB). In particular, ASB is widely observed at high strain rates and is considered to be a precursor to catastrophic failure. It usually involves thermal transients of large dynamic deformation, high-speed plasticity, endogenous heat, local temperature migration, and the local slip [1-3]. In the past decades, ASBs have been widely studied because of their importance in shock problems. Although many theoretical models have been developed [4-6] to understand the formation and structure of ASBs, there are still difficulties in providing insight into the evolution of complex ASBs.

Many experiments of ASBs have also been carried out and mostly are limited to metallurgical inspection of samples after the test. To track the evolution of the ASBs, Marchand and Duffy [7] and Zhou et al. [8] used linear arrays of infrared temperature detectors to measure the temperature distribution in the ASB. Guduru et al. [9] used a square array of 64 infrared temperature detectors to detect on a pre-notched plate. They found for the first time that the temperature field consists of a series of transient and periodic "hot spots" within the fully developed ASB.

\footnotetext{
* Corresponding authors.

E-mail addresses: liuzhanli@tsinghua.edu.cn (Z.L. Liu), zhuangz@tsinghua.edu.cn (Z. Zhuang).
} 
In order to reproduce more realistic experimental results, many efforts have been made to predict ASBs by developing twodimensional (2D) and three-dimensional (3D) numerical models. More complicated constitutive models based on damage mechanics have been adopted [10-13], which generally requires a specific criterion to decide the initiation and propagation of the dynamics ASBs. These models are mainly based on the evolution of local damage variables and can reflect the evolution of ASBs as a whole. However, the width of ASBs (depending on the mesh size) cannot be accurately characterized.

On the other hand, phase-field method (or energy variational method) has attracted much attention in recent years [14-17], due to its ability to describe the material failure, dislocation evolution, phase transition and diffusion crack propagation in the same framework. At present, the phase-field method has been widely used in investigating crack growth, merging, bifurcation, and other problems. It exhibits excellent prediction abilities, especially in multi-field coupled crack growth problems [18,19]. Miehe et al. $[18,20]$ developed the phase-field method for multi-physical problems and applied it to coupled problems such as thermal fracture and hydraulic fracturing. Duda et al. [21] proposed a phase-field model for brittle fracture of elastic-plastic solids containing mobile interstitial solute species. Geelen et al. [22] extended a phase field/gradient damage formula for the cohesive fracture to the dynamic problems. Wu et al. [23] extended the phase-field regularized cohesive zone model (PF-CZM) to deal with hydrogen assisted cracking. Recently, the potential ability of the phase-field method in simulating ASBs has attracted more attention [24-26]. They successfully reproduced single adiabatic shear bands by the phase-field method.

Though ASBs have been successfully simulated by the phase-field method, how to calibrate and select the material parameters in the phase-field model is seldom clearly discussed, even though it plays a dominated role in simulating fracture and failure problems. The lack of such work is because, in such problems with strong localization, the overall response of the specimen is very different from the mechanical behavior of the local material point. It is very challenging to obtain the damage parameters of the material by macroscopic material experiments, such as uniaxial tension or pure shear. For brittle or quasi-brittle materials, Feng et al. [27-29] proposed a quasi-micromechanical model of microcrack-weakened damage, which links the macroscopic response of materials with microstructure such as microcrack. Zeng et al. [30,31] further extended it to the dynamic high-speed impact problem of brittle ceramics. For materials with large plastic deformation, most of the previous work calibrate various damage and constitutive models, only through fitting the macroscopic response of the material [32-34].

In this paper, a thermo-elastic-plastic phase-field damage model is established to simulate the generation and evolution of ASBs, as well as its transition to fracture. The Johnson-cook (JC) model including strain hardening, strain rate hardening and thermal softening is adopted to describe the yield of materials. The damage parameters in the phase-field method are calibrated using data from pure shear specimens, taking into account both the overall response of the structure and the local response in the shear band. The established model can quantitatively characterize and predict the width of the ASB, the temperature in the ASB and the damage evolution process.

The paper is organized as follows: The basic principle and governing equations of the thermo-elastic-plastic phase-field model are presented in Section 2. In Section 3, the parameters $w_{0}$ and $g_{c s}$ are calibrated by experimental results. Then in Section 4, we use the developed model to study the transition process from ASB to fracture. The concluding remarks are given in Section 5 .

\section{The thermo-elastic-plastic phase-field model}

In the phase-field method, a damage variable $d \in[0,1]$ is introduced to describe the failure of the material. In particular, regions with $d=0$ and $d=1$ correspond to unbroken and fully broken states of the material. In this section, we propose a framework of the thermo-elastic-plastic phase-field model. The model consists of macro and micro force balance equation, energy balance equation and irreversible damage condition. In addition, the non-linear constitutive relation is also needed.

\subsection{Thermo-elastic-plastic damage model}

Consider the evolution of the deformation, temperature and fracture fields of an isotropic thermo-elastic plastic solid body $\Omega \subset \mathbb{R}^{\delta}$ with external boundary $\partial \Omega \subset \mathbb{R}^{\delta-1}$ over a time period [0- $\left.t_{f}\right]$, as shown in Fig. 1 . Herein, $\delta \in\{1,2,3\}$ is the dimension. Let $\partial \Omega_{t}, \partial \Omega_{\boldsymbol{u}}, \partial \Omega_{\boldsymbol{J}}$ and $\partial \Omega_{\theta}$ be the traction, displacement, heat flux and temperature boundaries, such that: $\partial \Omega_{\boldsymbol{t}} \cup \partial \Omega_{\boldsymbol{u}}=\partial \Omega, \partial \Omega_{\boldsymbol{t}} \cap \partial \Omega_{\boldsymbol{u}}=\varnothing$ and $\partial \Omega_{\boldsymbol{J}} \cup \partial \Omega_{\theta}=\partial \Omega, \partial \Omega_{\boldsymbol{J}} \cap \partial \Omega_{\theta}=\varnothing$. Let $\overline{\boldsymbol{t}}: \partial \Omega_{\boldsymbol{t}} \times\left[0, t_{f}\right] \rightarrow \mathbb{R}^{\delta}$ and $\overline{\boldsymbol{u}}: \partial \Omega_{\boldsymbol{u}} \times\left[0, t_{f}\right] \rightarrow \mathbb{R}^{\delta}$ be prescribed traction and displacement boundary conditions, and $\bar{\theta}: \partial \Omega_{\theta} \times\left[0, t_{f}\right] \rightarrow \mathbb{R}^{1}$ and $\bar{J}: \partial \Omega_{\boldsymbol{J}} \times\left[0, t_{f}\right] \rightarrow \mathbb{R}^{1}$ be prescribed temperature and heat flux boundary conditions.

According to the finite strain framework, the additive decomposition of the total rate of deformation $\boldsymbol{D}$ is assumed as follows:

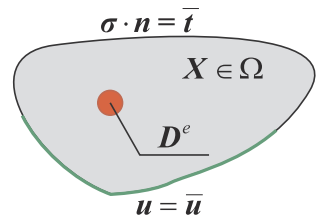

Deformation field

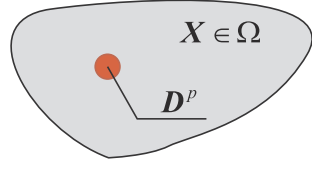

Internal variables

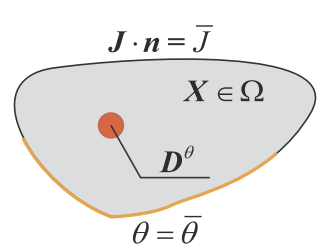

Temperature field

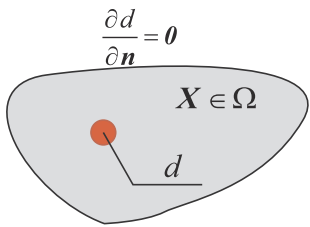

Phase field

Fig. 1. The main field variables of the thermo-elastic-plastic phase-field model. 


$$
\boldsymbol{D}=\boldsymbol{D}^{e}+\boldsymbol{D}^{p}+\boldsymbol{D}^{\ominus}
$$

where $\boldsymbol{D}^{e}, \boldsymbol{D}^{p}$ and $\boldsymbol{D}^{\ominus}$ are the elastic, plastic and thermal expansion parts of the deformation rate, respectively.

The thermo-elastic-plastic model is proposed based on a Cauchy stress $\sigma$ obtained from the damage-reduced inherent Cauchy stress of material $\sigma_{0}$. The elastic response defined in terms of the Green-Naghdi rate is specified by applying the hypoelastic law to the elastic deformation rate:

$$
\sigma=g(d) \sigma_{0}, \quad \Delta \sigma_{0}^{\nabla G}=\boldsymbol{C}_{0}^{\nabla G}: \boldsymbol{D}^{\boldsymbol{e}}
$$

where $\boldsymbol{C}_{0}^{\nabla G}$ is the inherent elastic stiffness tensor of material for Green-Naghdi rate, which is determined by Young's modulus $E$ and Possion's ratio $\nu . g(d)=(1-d)^{2}$ is the typical degradation function [35].

The isotropic hardening $J_{2}$ flow model of von Mises yield surface is adopted. This model is especially suitable for metal plasticity. The yield condition is

$$
f\left(\sigma ; \varepsilon_{p}, \dot{\varepsilon}_{p}, \theta, \cdots\right)=\sigma_{e q}(\sigma)-\sigma_{Y}\left(\varepsilon_{p}, \dot{\varepsilon}_{p}, \theta, \cdots\right)=0
$$

and the plastic deformation rate $\boldsymbol{D}^{p}$ is given as follows:

$$
\boldsymbol{D}^{p}=\dot{\lambda} \boldsymbol{r}, \quad \boldsymbol{r}=\frac{\partial f}{\partial \sigma}=\frac{3 \boldsymbol{S}}{2 \sigma_{e q}}, \quad \dot{\lambda}=\dot{\bar{\varepsilon}}_{p}
$$

where $\boldsymbol{r}$ is the plastic flow direction (associative plasticity). $\boldsymbol{S}$ is the deviatoric part of Cauchy stress, that is $\boldsymbol{S}=\frac{1}{3} \operatorname{trace}(\sigma) \boldsymbol{I}$. $\sigma_{e q}=\sqrt{3(\boldsymbol{S}: \boldsymbol{S}) / 2}$ is the equivalent mises stress. $\sigma_{Y}$ is the yield stress. $\dot{\boldsymbol{\varepsilon}}_{p}=\sqrt{2\left(\boldsymbol{D}^{p}: \boldsymbol{D}^{p}\right) / 3}$ is the equivalent plastic strain rate. $\varepsilon_{p}=\int \dot{\varepsilon}_{p} d t$ is the equivalent plastic strain.

The Johnson-cook (JC) model is used to describe the yield of materials, which considers the strain hardening, strain rate hardening and thermal softening effects:

$$
\sigma_{Y}^{0}=\left(A+B \varepsilon_{p}^{N}\right)\left(1+C \ln \frac{\dot{\varepsilon}_{p}}{\dot{\varepsilon}_{0}}\right)\left(1-\left(\frac{\theta-\theta_{0}}{\theta_{m}-\theta_{0}}\right)^{m}\right)
$$

where $\sigma_{Y}^{0}$ is the yield stress of the non-damaged material, $A, B, C, N$ and $m$ are the material parameters, $\theta_{m}$ is the melting temperature and $\theta_{0}$ is the reference temperature, $273 \mathrm{~K}$ here. $\dot{\varepsilon}_{0}$ is a reference strain rate, taken to be $\dot{\varepsilon}_{0}=1.0 \mathrm{~s}^{-1}$. When the material is damaged, the yield stress $\sigma_{Y}$ also deteriorates, and the degradation function is $g(d)$. In other words, $\sigma_{Y}=g(d) \sigma_{Y}^{0}$. is:

Considering isotropic thermal expansion, the thermal deformation rate $\boldsymbol{D}^{\ominus}$ in Eq. (1) is simplified. The thermal deformation rate

$$
\boldsymbol{D}^{\ominus}=\alpha_{\theta} \dot{\theta} \boldsymbol{I}
$$

where $\alpha_{\theta}$ is the thermal expansion coefficient of materials, and $\boldsymbol{I}$ is the identity tensor.

\subsection{Specification of the free energy}

The constitutive relationship now depends on the specific forms of the contribution of elasticity, plasticity, heat and fracture to free energy density. We use a general form in which the free energy density is decomposed into stored deformation (elastic and plastic), thermal and fracture parts: $\psi^{s}, \psi^{\ominus}$ and $\psi^{f}$, respectively.

$$
\psi=\psi^{s}+\psi^{\ominus}+\psi^{f}
$$

The storage deformation energy of materials consists of two parts: elastic part and plastic part. For intact materials (without failure), the elastic deformation energy density and plastic deformation energy density that drive damage evolution are:

$$
\begin{aligned}
& \psi_{e 0}=\int_{0}^{t} \sigma_{0}: \boldsymbol{D}^{e} \mathrm{~d} \tau \\
& \psi_{p 0}=\int_{0}^{t}(1-\chi) \sigma_{0}: \boldsymbol{D}^{p} \mathrm{~d} \tau
\end{aligned}
$$

where $\chi \in(0,1]$ is the fraction of plastic work converted to heat. The remaining plastic work is used to generate new fracture surfaces. $\chi$ is usually considered as a constant and independent the material. In this paper, we assume it as 0.9 [24,25,36].

The elastic strain energy density is usually decomposed into two parts according to the contribution to the driving of the fracture phase field: $\psi_{e 0}=\psi_{e 0}^{-}+g(d) \psi_{e 0}^{+}$. The example of $\psi_{e 0}^{+}$used in small strain analysis in literature is volumetric "deviatoric split of strain energy density. In this split, the tensile volumetric and deviatoric strains contribute to the damage phase field [37,38]:

$$
\psi_{e 0}^{+}=(\lambda / 2+\mu / 3)\left\langle\operatorname{tr} \varepsilon^{e}\right\rangle_{+}^{2}+\mu\left\|\operatorname{dev} \varepsilon^{e}\right\|^{2}
$$

where $\varepsilon^{e}$ is the small elastic strain tensor, $\operatorname{tr} \varepsilon^{e}$ and $\operatorname{dev} \varepsilon^{e}$ are the volumetric and deviatoric parts of it, $\lambda$ and $\mu$ are Lame's constants, and $\langle a\rangle:=(a \pm|a|) / 2$ for any $a \in \mathbb{R}$.

Another popular split, used in Refs. $[35,39,40]$, is based on the spectral decomposition of the small strain tensor, so that 


$$
\psi_{e 0}^{+}=(\lambda / 2)\left\langle\operatorname{tr} \varepsilon^{e}\right\rangle_{+}^{2}+\mu \sum_{i=1}^{3}\left\langle\epsilon_{i}^{e}\right\rangle_{+}^{2}
$$

where $\epsilon_{i}^{e}$ are the principal strains. In this split, only pure tensile deformation contributes to the damage phase field.

Recently, some new decomposition methods have been proposed to deal with more complex problems. For example, Wu et al. $[41,42]$ proposed a phase-field anisotropic damage model based on the unified phase-field damage theory [43,44]. The unilateral effect is treated by the positive/negative projection of the effective stress in the energy specification, which is suitable for brittle fracture and quasi-brittle failure. In all the simulations in this paper, the volumetric deviatoric split method is used to consider the resistance of materials to hydrostatic pressure and is consistent with plastic yield (hydrostatic pressure does not cause yielding).

To better modeling the damage initiation, we introduce the energy density threshold $w_{0}$. It can be understood as the storage deformation energy required for the microstructure evolution before the damage initiation, such as recrystallization. Only the sum of $\psi_{e 0}^{+}$and plastic energy storage except $w_{0}$ contributes to damage evolution and is degraded by function $g(d)=(1-d)^{2}$. Therefore, the stored deformation energy density can be expressed as:

$$
\psi^{s}=\psi_{e 0}^{-}+\min \left[\psi_{e 0}^{+}+\psi_{p 0},(1-\chi) w_{0}\right]+g(d)\left\langle\psi_{e 0}^{+}+\psi_{p 0}-(1-\chi) w_{0}\right\rangle_{+}
$$

Let the specific heat $c$ be a constant, and the thermal part of the free energy density is given [45]:

$$
\psi^{\theta}=-\rho c \theta \ln \frac{\theta}{\theta_{0}}
$$

where $\rho$ is the mass density.

The fracture energy density is given by the classical phase field theory $[46,24]$

$$
\psi_{f}=\frac{g_{c s}}{2 l_{c}}\left(d^{2}+l_{c}^{2}\|\nabla d\|^{2}\right)
$$

where $g_{c s}$ is the effective critical energy release rate, $l_{c}$ is a length scale parameter that can characterize crack width. We will later explain that in the evolution of the ASBs of metallic materials, $g_{c s}$ and $w_{0}$ are essential for the damage evolution, and will be explained in detail in Section 3.

\subsection{Governing equations}

The solution domain of the elastic-plastic fracture problem considering the evolution of temperature and phase field is as follows

$$
\begin{aligned}
\mathcal{T}^{\boldsymbol{u}} & :=\left\{\boldsymbol{u} \in H^{1}\left(\Omega ; \mathbb{R}^{\delta}\right) \times\left[0, t_{f}\right] \mid \boldsymbol{u}(\cdot, t)=\overline{\boldsymbol{u}}(\cdot, t) \text { on } \partial \Omega_{\boldsymbol{u}}\right\}, \\
\mathcal{T}^{\theta} & :=\left\{\theta \in H^{1}\left(\Omega ; \mathbb{R}^{1}\right) \times\left[0, t_{f}\right] \mid \theta(\cdot, t)=\bar{\theta}(\cdot, t) \text { on } \partial \Omega_{\theta}\right\}, \\
\mathcal{T}^{d} & :=\left\{d \in H^{1}(\Omega ;[0,1]) \times\left[0, t_{f}\right]\right\},
\end{aligned}
$$

Then the regularized variational formulation reads: Find $(\boldsymbol{u}, \theta, d) \in \mathcal{T}^{\boldsymbol{u}} \times \mathcal{T}^{\theta} \times \mathcal{T}^{d}$ that is the stationary point of $S_{l_{c}}[\boldsymbol{u}, \dot{\boldsymbol{u}}, \theta, \Gamma]:=\int_{0}^{t_{f}} L_{l_{c}}[\boldsymbol{u}, \dot{\boldsymbol{u}}, \theta, \Gamma] d t$, with

$$
\begin{aligned}
L_{l_{c}}[\boldsymbol{u}, \dot{\boldsymbol{u}}, \theta, \Gamma]:= & \int_{\Omega}\left\{\frac{1}{2} \rho \dot{\boldsymbol{u}} \cdot \dot{\boldsymbol{u}}-\psi(\boldsymbol{D}(\boldsymbol{u}, \theta), d)+\rho \boldsymbol{b} \cdot \boldsymbol{u}+\rho c \dot{\theta}+\nabla \cdot \boldsymbol{J}-\gamma\right\} d V+\int_{\partial \Omega_{\boldsymbol{t}}} \overline{\boldsymbol{t}} \cdot \boldsymbol{u} d \Gamma+\int_{\partial \Omega_{\boldsymbol{J}}} \overline{\boldsymbol{J}} \cdot \boldsymbol{n} d \Gamma-\frac{g_{c s}}{2 l_{c}} \\
& \int_{\Omega}\left(d^{2}+l_{c}^{2}\|\nabla d\|^{2}\right) d V
\end{aligned}
$$

where $\boldsymbol{b}$ is the body force vector per unit mass, $\gamma$ is the body heat source per unit volume, $\Gamma$ is the set of discontinuities and $\boldsymbol{J}$ is the internal heat flux which is assumed to be proportional to the temperature gradient: $\boldsymbol{J}=-k \cdot \nabla \theta$. Here $k$ is the thermal conductivity. The strong form of the boundary value problem can be derived from Eq. (16):

$$
\begin{aligned}
& \operatorname{div} \sigma+\rho \boldsymbol{b}=\rho \ddot{\boldsymbol{u}} \text { in } \Omega \times\left[0, t_{f}\right] \\
& \rho c \dot{\theta}+\nabla \cdot \boldsymbol{J}=\gamma \quad \text { in } \Omega \times\left[0, t_{f}\right] \\
& \frac{g_{c s}}{2 l_{c}}\left(d-l_{c}^{2} \Delta d\right)=(1-d)\left\langle\psi_{e 0}^{+}+\psi_{p 0}-(1-\chi) w_{0}\right\rangle \text { in } \Omega \times\left[0, t_{f}\right] \\
& \sigma \cdot \boldsymbol{n}=\overline{\boldsymbol{t}} \quad \text { on } \quad \partial \Omega_{\boldsymbol{t}} \times\left[0, t_{f}\right] \\
& \boldsymbol{J} \cdot \boldsymbol{n}=\bar{J} \quad \text { on } \quad \partial \Omega_{J} \times\left[0, t_{f}\right] \\
& \frac{\partial d}{\partial \boldsymbol{n}}=0 \quad \text { on } \quad \partial \Omega \times\left[0, t_{f}\right]
\end{aligned}
$$

In order to satisfy the condition of irreversible damage, we introduce a local historical field variable $\mathcal{H}$ to record the historical maximum of phase field driving energy density: 


$$
\mathcal{H}(\boldsymbol{x}, t)=\max _{s \in[0, t]}\left\langle\psi_{e 0}^{+}(\boldsymbol{x}, s)+\psi_{p 0}(\boldsymbol{x}, s)-(1-\chi) w_{0}(\boldsymbol{x}, s)\right\rangle
$$

Thus the evolution equation of the phase field can be rewritten as follows

$$
\frac{g_{c s}}{2 l_{c}}\left(d-l_{c}^{2} \Delta d\right)=(1-d) \mathcal{H} \quad \text { in } \quad \Omega \times\left[0, t_{f}\right]
$$

The above formula determines the evolution of damage phase-field. It can be seen that $\mathcal{H}$ acts as the driving force, $g_{c s}$ acts as the "damping" of evolution, and $l_{c}$ is located in front of the diffusion term, which represents the diffusion of damage to the surrounding area and has a very important influence on the evolution of the width of the shear band.

\subsection{Rate-dependent form of phase-field evolution}

In order to adopt explicit time integral, a time-dependent form according to Ref. $[14,15,35]$ is used to determine the phase field evolution:

$$
\dot{d}= \begin{cases}\frac{1}{\eta}\left\langle(1-d) \mathcal{H}-\frac{g_{c s}}{2 l_{c}}\left(d-l_{c}^{2} \Delta d\right)\right\rangle_{+}, & d<1 \\ 0, & \text { otherwise }\end{cases}
$$

where $\eta$ is the viscous parameter. It has an important influence on the evolution of phase field, which has been discussed in detail in our previous articles [14,38].

The initial condition of the problem can be expressed as

$$
\boldsymbol{u}(\cdot, 0)=\boldsymbol{u}_{0}, \quad \dot{\boldsymbol{u}}(\cdot, 0)=\boldsymbol{v}_{0}, \quad \theta(\cdot, 0)=\theta_{0}, \quad d(\cdot, 0)=d_{0},
$$

where $\boldsymbol{u}_{0}, \boldsymbol{v}_{0}, \theta_{0}$ and $d_{0}$ are the initial displacement, velocity, temperature and damage field, respectively. The above phase-field model is implemented in the commercial finite element software package Abaqus/Explicit by writing the user subroutine VUEL and VUMAT. Details of the implementation can be found in our previous article [14,38].

\section{Parametric calibration of $w_{0}$ and $g_{c s}$}

In the phase-field model proposed in Section 2, there are two main unknown parameters: $w_{0}$ and $g_{c s}$. Among them, $w_{0}$ determines the energy density required for damage initiation, while $g_{c s}$ determines the speed of damage evolution. In the following, we will firstly demonstrate the effect of these two parameters through element testing, and then present the method of determining their values according to experimental data.

\subsection{Element testing}

In order to investigate more clearly the effects of $w_{0}$ and $g_{c s}$ on the response of materials and structures at high strain rates, we firstly tested the uniaxial tension of different materials at different strain rates, $w_{0}$ and $g_{c s}$. Note that the element test is performed here, and hence the local stress-strain response at the material point is obtained. It is different from the overall response of the structure and the macroscopic stress-strain curve we usually get from the experiment. To keep the strain rate of each uniaxial tension constant, considering the large deformation of the structure, the axial velocity of uniaxial tension is $v=L_{0} \dot{\varepsilon}_{c} e^{\dot{\varepsilon}_{c} t}$. Where $L_{0}$ is the initial axial length of the uniaxial tensile test model, $\dot{\varepsilon}_{c}=$ constant is target axial strain rate. The material parameters used in the present study are listed in Tables 1 and 2. The viscous parameter $\eta$ is taken as $\eta=5.0 \times 10^{-8} \mathrm{kN} * \mathrm{~s} / \mathrm{mm}^{2}$. For more information on the effects of viscous parameter on the results, see our previous articles [14]. In Fig. 2, the stress-strain curves of three materials ((a) Ss304L; (b) Cu and (c) Ti6Al4V) under uniaxial tension test with one element are given.

For the same material (such as Ss304L in Fig. 2(a)), the same $w_{0}$ will lead to the same peak stress and initial softening strain. The value of $w_{0}$ is the strain energy density stored in the material from the free state to the peak stress state. The value of $g_{c s}$ determines the speed of stress drop (i.e. the speed of damage evolution). The larger the value of $g_{c s}$, the slower the damage evolution. The response of Ti6Al4V is similar, except that the falling portion of the stress-strain curve is more "steep" and the strain at the beginning of the damage is smaller, as shown in Fig. 2(c). Therefore, Ti6Al4V is more brittle, compared with Ss304L and Cu.

We also validate our code by comparing it with the JC plastic model built in Abaqus, as shown in Fig. 2(a). It can be seen that in

\begin{tabular}{|c|c|c|c|c|c|c|c|c|c|}
\hline & $\rho_{0}\left[\mathrm{~kg} / \mathrm{m}^{3}\right]$ & $E$ [GPa] & $v$ & $A[\mathrm{GPa}]$ & $B$ [GPa] & C & $N$ & $m$ & $\theta_{\mathrm{m}}\left[{ }^{\circ} \mathrm{C}\right]$ \\
\hline Ss304L & 7900.0 & 210.0 & 0.29 & 0.1 & 1.072 & 0.05 & 0.34 & 1.0 & 1083.0 \\
\hline Ti6Al4V & 4430.0 & 110.0 & 0.33 & 0.862 & 0.331 & 0.012 & 0.34 & 0.8 & 1630.0 \\
\hline $\mathrm{Cu}$ & 8920.0 & 120.0 & 0.2 & 0.09 & 0.292 & 0.025 & 0.31 & 1.09 & 1063.0 \\
\hline HY100 Steel & 7746.0 & 207.0 & 0.3 & 0.758 & 0.402 & 0.011 & 0.26 & 1.13 & 1500.0 \\
\hline
\end{tabular}
the ascending segment of the stress-strain curve, our model agrees well with the program calculations built in Abaqus. Since the JC

Table 1

Material parameters of JC model for metals $[13,47,48]$. 
Table 2

Other material parameters for metals $[13,47,48]$.

\begin{tabular}{|c|c|c|c|c|c|}
\hline & $k\left[\mathrm{~W} / \mathrm{m}^{\circ} \mathrm{C}\right]$ & $c\left[\mathrm{~J} / \mathrm{kg}^{\circ} \mathrm{C}\right]$ & $\alpha_{\theta}\left[10^{-6} /{ }^{\circ} \mathrm{C}\right]$ & $g_{c s}[\mathrm{~N} / \mathrm{mm}]$ & $w_{0}\left[\mathrm{~J} / \mathrm{mm}^{3}\right]$ \\
\hline Ss304L & 16.2 & 500.0 & 17.3 & 2.0 & 0.15 \\
\hline Ti6Al4V & 6.6 & 670.0 & 9.0 & 1.0 & 0.14 \\
\hline $\mathrm{Cu}$ & 147.0 & 380.0 & 16.7 & 1.2 & 0.105 \\
\hline HY100 Steel & 34.0 & 502.0 & 14.0 & 8.0 & 0.33 \\
\hline
\end{tabular}

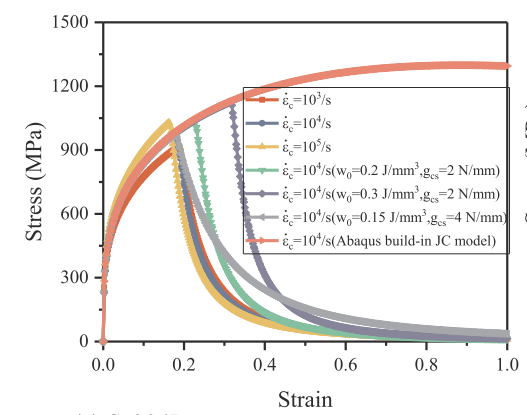

(a) Ss304L

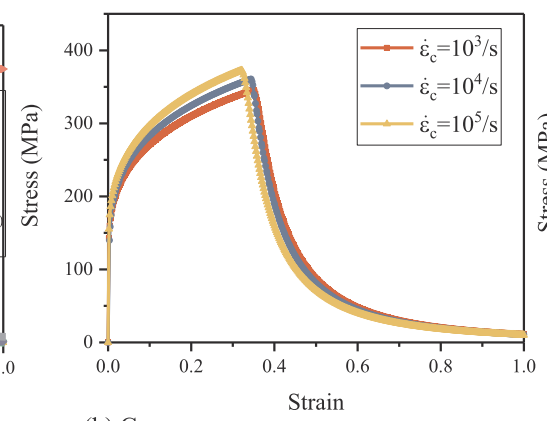

(b) $\mathrm{Cu}$

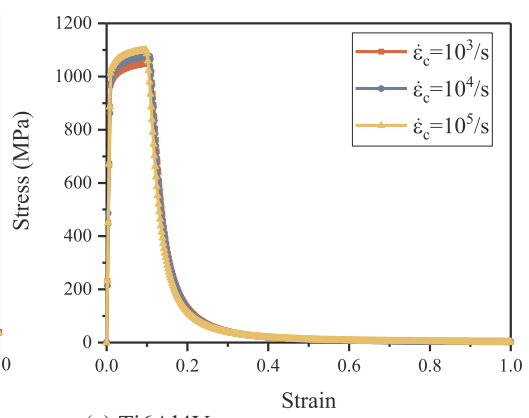

(c) Ti6Al4V

Fig. 2. The stress-strain curves of the three materials at different strain rates, $w_{0}$ and $g_{c s}$ : (a) Ss304L; (b) Cu and (c) Ti6Al4V, as well as the comparison of Abaqus built-in JC model.

plastic model built in Abaqus does not consider the damage effect, there is no softening regime. However, our algorithm can well capture both the hardening stage of JC plastic model and damage softening of the metal.

\subsection{Pure shear test and parameter calibration}

As we pointed out earlier, the material model is the stress-strain response at the local material point (input), while the response of the whole structure is obtained in the experiment (output). Because of the high localization of the shear band, the response of the material in the shear band is quite different from that of the whole structure, so the whole response of the structure cannot be directly used to represent the local response of the material. The reasonable model input parameters, especially $w_{0}$ and $g_{c s}$, can be determined by one-to-one mapping between them. Here, we take HY100 steel as an example and calibrate the whole shear stress-shear strain curve of pure shear test of Marchand and Duffy [7] to obtain reasonable parameters of local material points. The pure shear model is realized by torsion of thin-walled cylinder. The geometric and load diagrams of the model are shown in Fig. 3. A defect was added to the center of the cylinder to specify the position of the shear band initiation. The minimum mesh size of the model is 0.01 mm, which is much smaller than the length scale parameter $l_{c}=0.1 \mathrm{~mm}$.

The overall distribution of damage and temperature are presented in Fig. 4(a) and (b). The nominal strain rate is $1600 \mathrm{~s}^{-1}$. It can be seen that in the middle of the model, an ASB is formed across the whole ring. The maximum temperature in the shear band is $244.45^{\circ} \mathrm{C}$. The local deformation of the shear band under several nominal shear strains is shown in Fig. 4(c)-(g). Initially, the shear deformation on the whole model is homogeneous. When the nominal shear strain reaches a certain value (as $\gamma_{\text {NOM }}=0.47$ in Fig. 3 ), shear localization occurs near the defect and rapidly expands into a a circular shear band. Afterwards, severe shear localization occurs with loading. The area outside the shear band keeps uniform deformation, and the width of the shear band will not continue to increase with the loading.

Fig. 5 shows the response curves of the whole structure and the local response in the shear band, and compares them with the

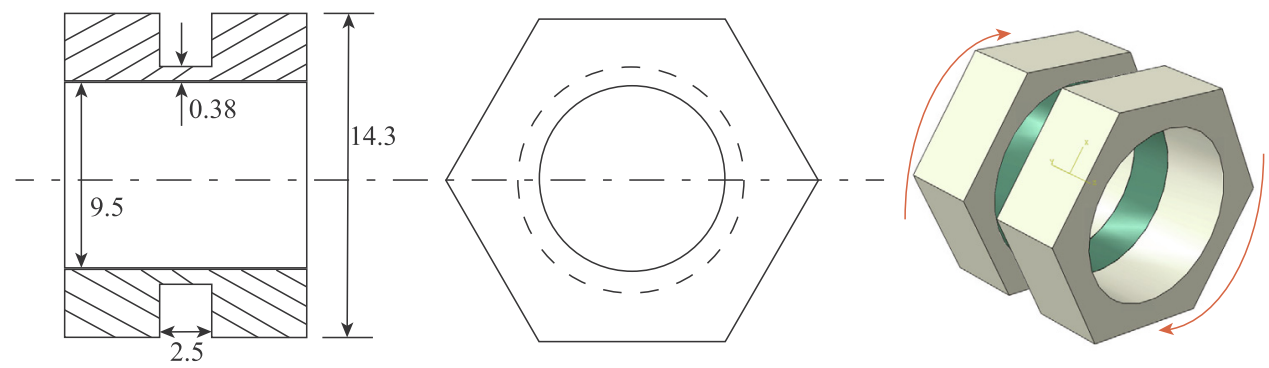

Fig. 3. Detailed information of torsion specimen of the hexagonal flange, including geometric dimensions and load diagram [7]. All length dimensions are in $\mathrm{mm}$. 


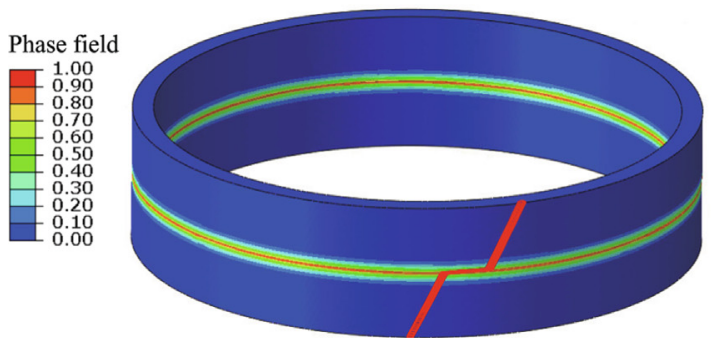

(a)Damage distribution at $\gamma_{\text {NOM }}=67 \%$

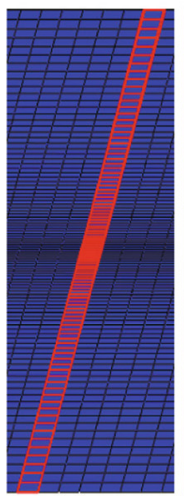

(c) $\gamma_{\mathrm{NOM}}=25 \%$

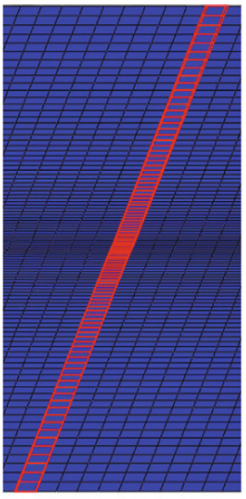

(d) $\gamma_{\text {NOM }}=36 \%$

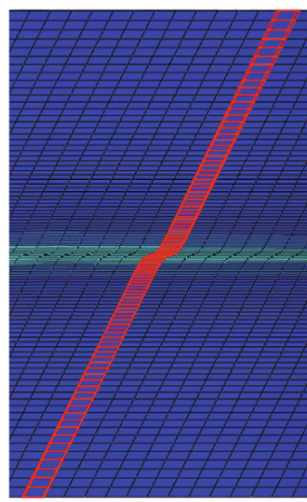

(e) $\gamma_{\mathrm{NOM}}=47 \%$

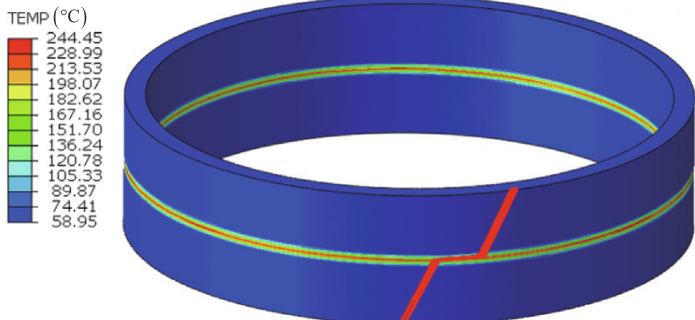

(b) Temperature distribution at $\gamma_{\mathrm{NOM}}=67 \%$

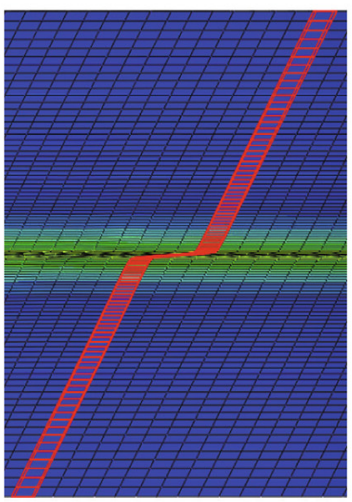

(f) $\gamma_{\text {NOM }}=55 \%$

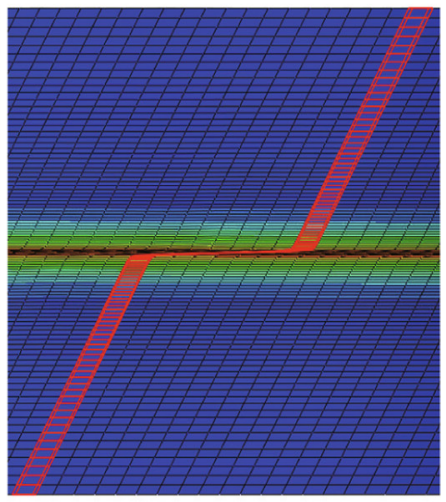

(g) $\gamma_{\text {NOM }}=67 \%$

Fig. 4. The simulation results of pure shear cases. The overall distribution of (a) damage and (b) temperature, and the local deformation under several shear strains are presented: (c) $\gamma_{\mathrm{NOM}}=0.25$, (d) $\gamma_{\mathrm{NOM}}=0.36$, (e) $\gamma_{\mathrm{NOM}}=0.47$, (f) $\gamma_{\mathrm{NOM}}=0.55$, (g) $\gamma_{\mathrm{NOM}}=0.67$. The nominal strain rate is $1600 \mathrm{~s}^{-1}$.

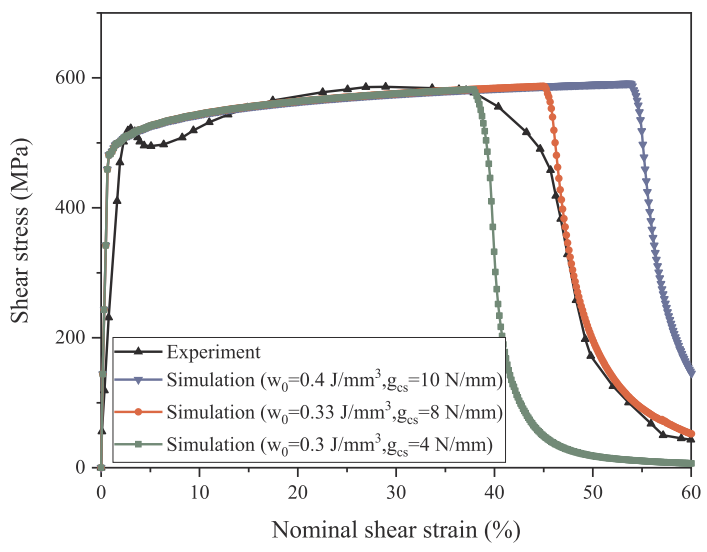

(a) Global response: shear stress - strain curve

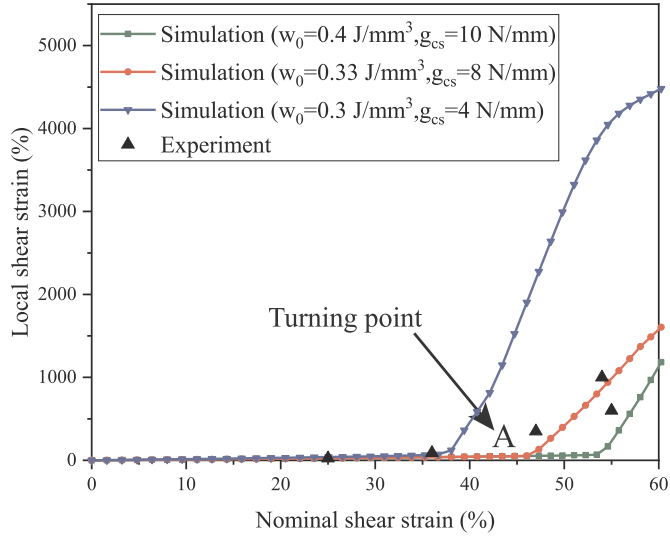

(b) Local response:shear strain in ASB - global strain

Fig. 5. Calibration process of parameters $w_{0}$ and $g_{c s}$. (a) The global stress-strain curves under different $w_{0}$ and $g_{c s}$ and their comparison with experiment results; (b) shear strain curves in local shear band versus global shear strain. The experiment results are obtained from [7].

experimental results. By choosing the appropriate $w_{0}$ and $g_{c s}$ (in this case, $w_{0}=0.33 \mathrm{~J} / \mathrm{mm}^{3}, g_{c s}=8.0 \mathrm{~N} / \mathrm{mm}$ ), the stress-strain curve of the whole structure and the local response in the shear band can be in good agreement with the experimental results. Especially in the softening section of the overall stress-strain curve, our model can still be in good agreement with the experimental values. At this stage, the ASB evolves rapidly. This shows that it is reasonable to take $w_{0}$ and $g_{c s}$ as the parameters of damage and shear band evolution in this paper. In addition, through the local strain in shear band - nominal shear strain curve, the initial strain of the rapid localization of the shear band can be clearly observed (i.e., the turning point ' $A$ ' of the curve in Fig. 5(b)).

The width of ASB is an important measure of it. The shear band width predicted by the damage based method often depends on the mesh size. Here, we investigate the distribution of shear band width and phase-field value under different mesh size $h$ and different characteristic width $l_{c}$, as shown in Fig. 6 . It can be seen that the width of shear band is dependent on the length scale parameter $l_{c}$. Therefore, for the shear band problem, $l_{c}$ can be used as an intrinsic material parameter to describe the characteristic width of the adiabatic shear band. 

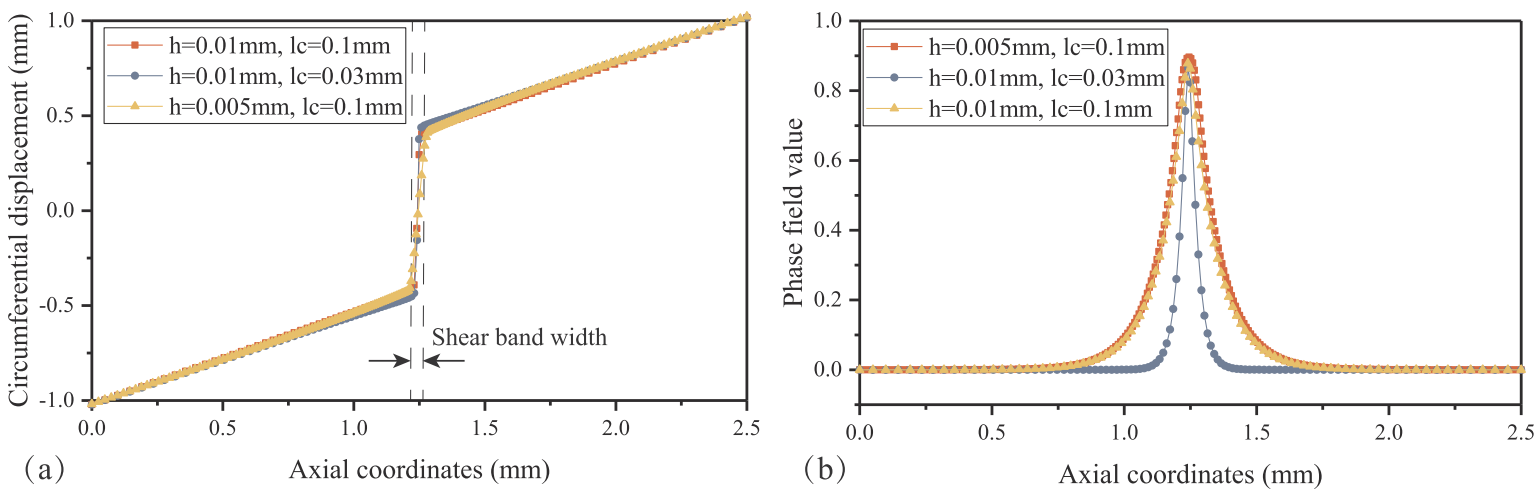

Fig. 6. Simulation of pure shear process with different feature width $l_{c}$ and mesh size $h$. (a) The curve of the circumferential displacement changing with the axial coordinate, and the place where the displacement suddenly jumps is the ASB; (b) the curve of phase-field value changing with axial coordinate.

\section{Application in the transition from ASB to fracture}

In this section, we use the impact test of the hat specimen as the application of the model, and further study the transition from ASB to fracture. Tungsten alloys are often used as kinetic energy penetrators because of their high density and strength. Under compression, ASB is the main failure mode of tungsten alloy. The further study of ASB is helpful to improve the performance of tungsten alloy armor piercing projectile.

The test is carried out by split Hopkinson pressure bar, and its schematic diagram is shown in Fig. 7(a). The specimen was placed on a $448 \mathrm{~mm}$ long, $20 \mathrm{~mm}$ diameter Hopkinson pressure bar. A $90 \mathrm{~mm}$ long and $20 \mathrm{~mm}$ diameter striker bar was fired with a gas gun. The impact speed range is $20-50 \mathrm{~m} / \mathrm{s}$ to ensure elastic deformation of the pressure bar and striker bar. In this paper, the initial impact velocity is $27.6 \mathrm{~m} / \mathrm{s}$. The section shape and size of the hat specimen are shown in Fig. 7(b). The radii of the interior and exterior corners are $0.15 \mathrm{~mm}$ and $0.12 \mathrm{~mm}$ respectively. Because of the high singularity of the corner, the two corners act as the starting point of the ASB and the fracture, and the effect is the same as that of the notch tip of the pre-notched plate.

According to the experimental conditions, the finite element model as shown in Fig. 7(c) is established. To improve the calculation efficiency, 1/4 model and symmetry boundary condition are used. The simply supported boundary condition is adopted at the end of the pressure bar. The pressure bar, striker bar and hat specimen are all made of tungsten alloy, and the material parameters are shown in Table 3.

The simulated phase-field evolutions at different times are shown in Fig. 8. The isosurface with $d=0.99$ is shown in the figure to see the 3D fracture morphology more clearly. In this paper, we consider that the ASB with phase-field variable $d \geqslant 0.99$ is transformed into a fracture. At this time, the shear band can hardly bear the load. It can be found that with the evolution of the ASB, at two corners, the ASB first transforms into fracture. As the loading continues, the fracture at the interior corners propagates faster than that

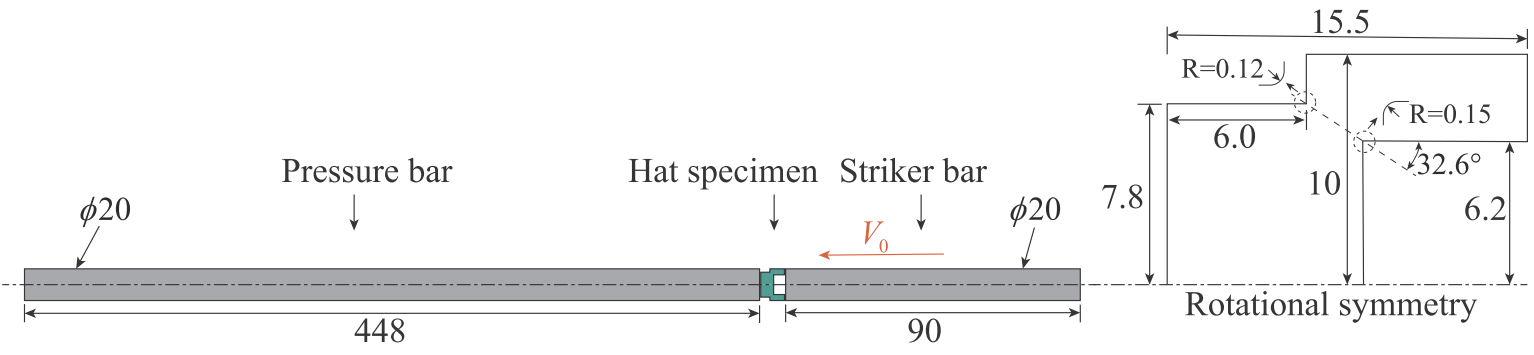

(a) Schematic diagram of impact test of hat specimen

(b) Section size of hat specimen

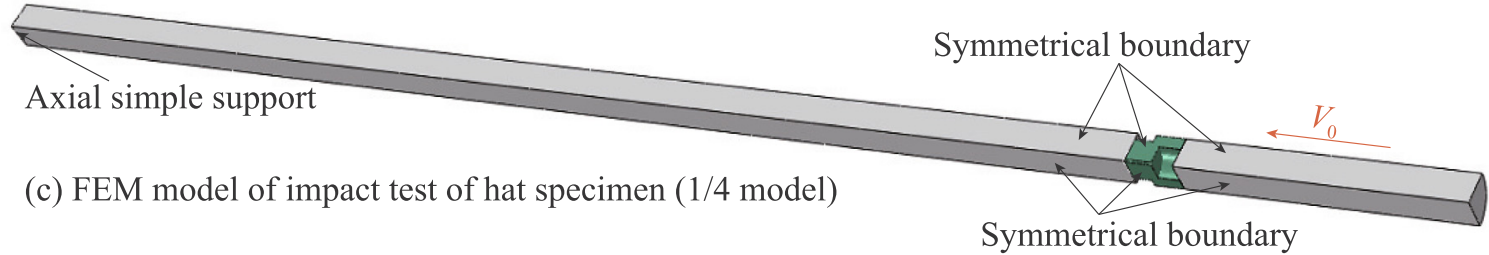

Fig. 7. Impact test of hat specimen: (a) schematic diagram of impact test of hat specimen, (b) section size of hat specimen (all length dimensions in the drawing are in mm) [49], and (c) FEM model of impact test of hat specimen (1/4 model). 
Table 3

Material parameters of JC model for tungsten alloys [49,50].

\begin{tabular}{|c|c|c|c|c|c|c|c|}
\hline$E[\mathrm{GPa}]$ & $v$ & $A[\mathrm{GPa}]$ & $B$ [GPa] & $C$ & $N$ & $m$ & $\theta_{\mathrm{m}}\left[{ }^{\circ} \mathrm{C}\right]$ \\
\hline 370.0 & 0.3 & 1.984 & 1.875 & 0.03 & 0.95 & 0.835 & 1085.0 \\
\hline$\rho_{0}\left[\mathrm{~kg} / \mathrm{m}^{3}\right]$ & $k\left[\mathrm{~W} / \mathrm{m}^{\circ} \mathrm{C}\right]$ & $c\left[\mathrm{~J} / \mathrm{kg}^{\circ} \mathrm{C}\right]$ & $\alpha_{\theta}\left[10^{-6} /{ }^{\circ} \mathrm{C}\right]$ & $g_{c s}[\mathrm{~N} / \mathrm{mm}]$ & $w_{0}\left[\mathrm{~J} / \mathrm{mm}^{3}\right]$ & & \\
\hline 17650.0 & 175.0 & 150.0 & 0.29 & 8.0 & 0.6 & & \\
\hline
\end{tabular}

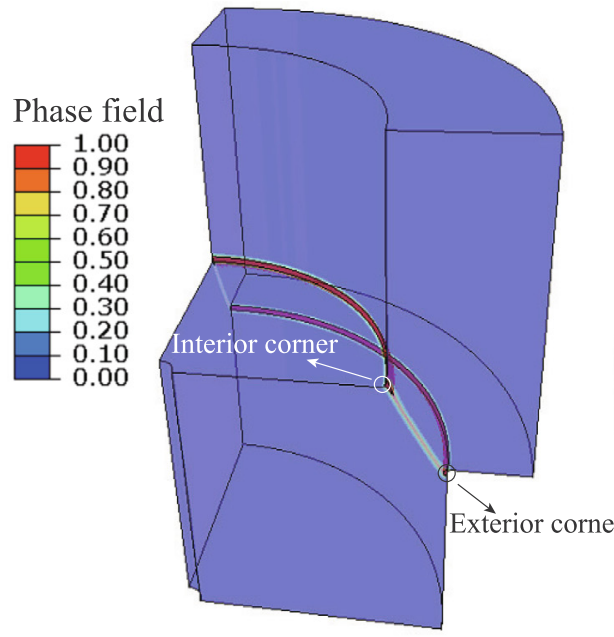

(a) $t=35 \mu \mathrm{s}$

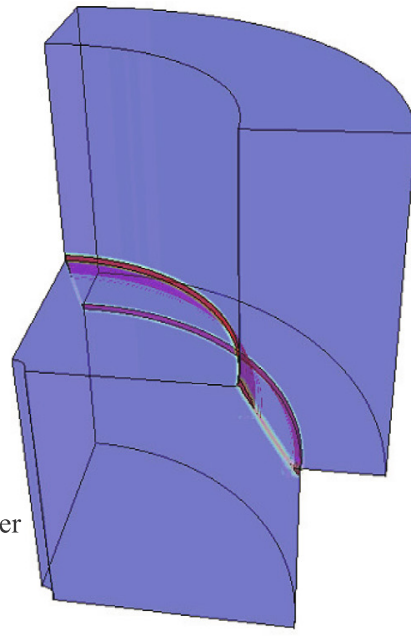

(b) $t=39 \mu \mathrm{s}$

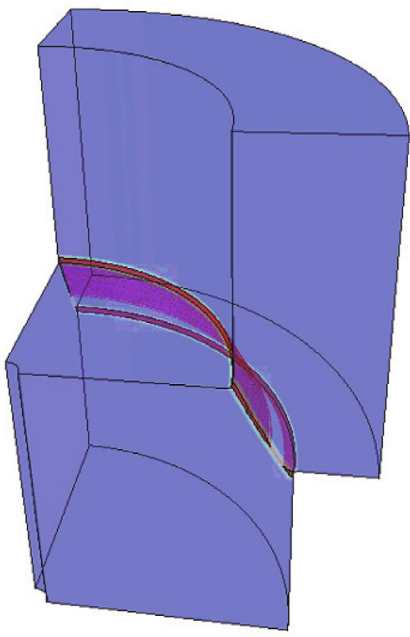

(c) $t=43 \mu \mathrm{s}$

Fig. 8. Distribution of ASBs simulated at different moments (the isosurface with $d=0.99$ is shown, and the view is transparent): (a) $t=35 \mu$; (b) $t=39 \mu \mathrm{s}$; and (c) $t=43 \mu \mathrm{s}$.

at the exterior corner. The final fracture does not penetrate the hat specimen. The total length of the fracture extending from the interior and exterior corner accounts for 0.7 times of the distance between the two corners. This is in good agreement with the previous simulation and experiment results, in which the measured length of the ASB is $71 \%$ of the gauge section at $V_{0}=27.6 \mathrm{~m} / \mathrm{s}$ $[49,50]$.

The temperature rise in the process of ASB's evolution is an important factor for its transformation into fracture. Temperature distributions across and along ASB are shown in Fig. 9. It can be found that there is a low temperature rise (about $200{ }^{\circ} \mathrm{C}$ ) before the ASB develops into a fracture. However, in the process of the transformation from the ASB into fracture, it is accompanied by the rapid rise of temperature. The temperature rise is about $100{ }^{\circ} \mathrm{C}$, which is close to the melting point of tungsten alloy. Fig. 9 (a) also shows that there is an overall temperature rise in the specimen near the striker bar, which indicates that the specimen enters into plasticity as a whole near the striker bar.

In addition, we also notice that the temperature distribution is not continuous, but there are some periodic discontinuous "hot spots". For the first time, Guduru et al. [9] found that the temperature field in the ASB consists of a series of transient and periodic "hot spots" fully developed along the ASB. This is different from the traditional continuous distribution of temperature field. Our simulation also shows that, as shown in Fig. 9(b), a series of "hot spots" are distributed in the ASB. These "hot spots" can be considered as the precursor of the transition of ASBs into fracture.

As we mentioned earlier, the width is an important measure of the ASB. In order to quantitatively study the width of ASB, we draw the distribution of displacement magnitude across ASB at different times in Fig. 10. The whole process from uniform deformation to shear localization, and then transition to fracture can be clearly seen from the figure. In the ASB, the displacement has an obvious mutation. The range of displacement jump is the width of ASB. This way of characterizing the width of the ASB is natural, which is more reasonable than the previous way of characterizing the width of the ASB by the temperature rise [49]. In their characterization, it is necessary to consider a given temperature rise as a material parameter. The width of the simulated ASB is $45 \mu \mathrm{m}$, which is in good agreement with the previous experimental results (about $40 \mu \mathrm{m}$ ) [49,50]. In addition, we can see that the width of the shear band does not continue to increase with the time evolution and the further increase of deformation in the shear band.

We also take a gauge point near the interior corner of the specimen and output the curves of stress, thermal softening factor and damage softening factor with time, as shown in Fig. 11. It is found that both thermal softening and damage softening have an important influence on the evolution of ASBs in tungsten alloy. Moreover, thermal softening precedes damage softening. Before damage softening, the yield strength of the material is reduced by about $10 \%$ due to thermal softening. In the later stage, the thermal softening and damage softening are carried out simultaneously, resulting in the rapid evolution and transformation of the ASBs into 

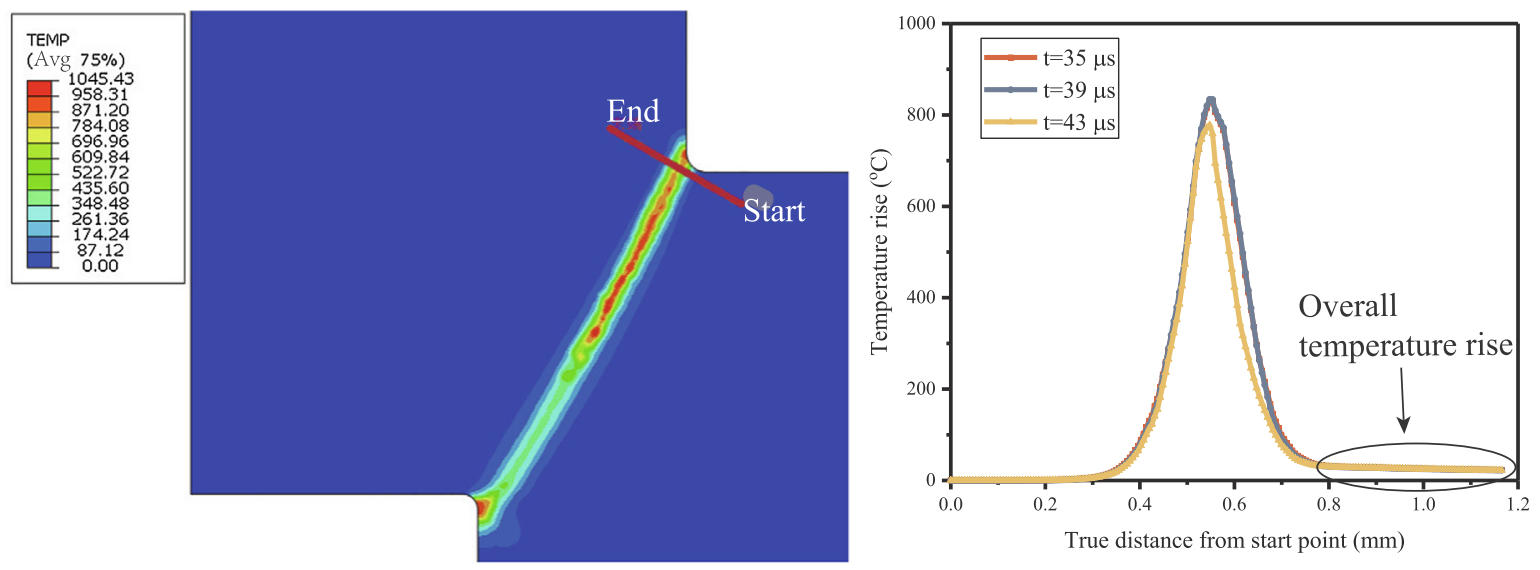

(a) The path across the shear band and its temperature rise distribution
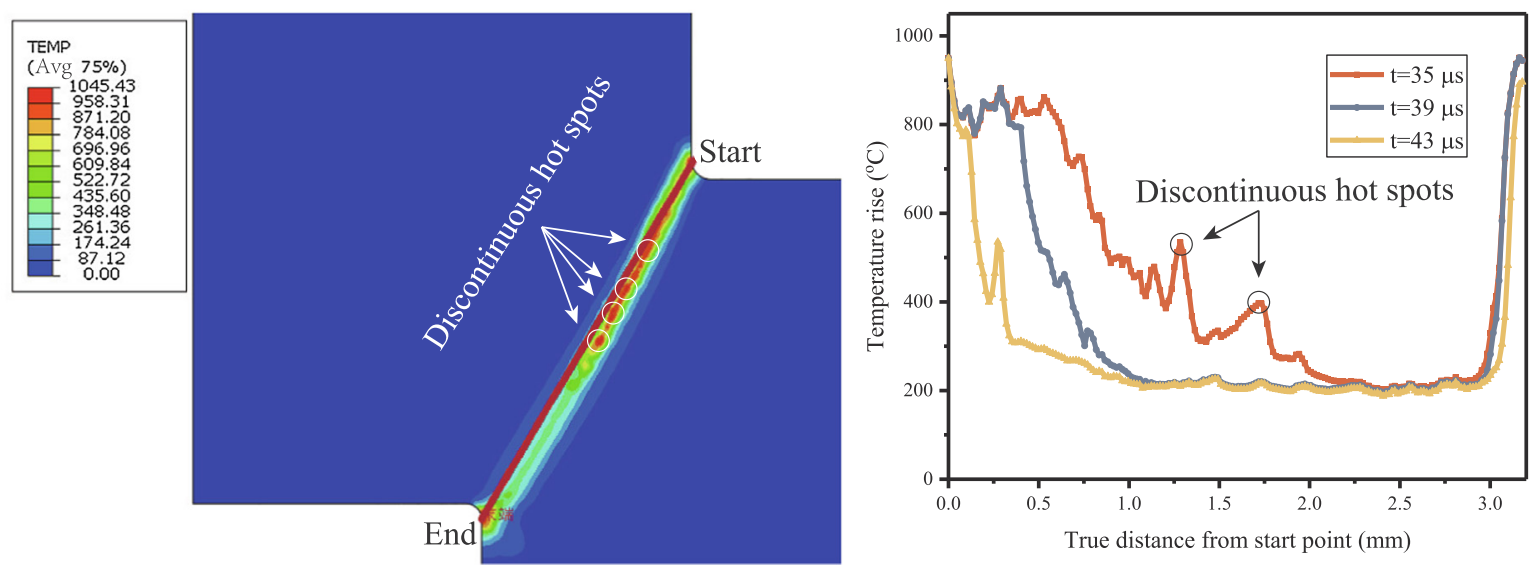

(b) The path along the shear band and its temperature rise distribution

Fig. 9. Temperature distributions across and along ASB: (a) the path across the shear band (left) and its temperature rise distribution (right), and (b) the path along the shear band (left) and its temperature rise distribution (right).

fractures. That is, two softening mechanisms work together. We call it double softening. After the joint action of thermal softening and damage softening, the stress at the gauge point decreased to nearly 0 at about $30 \mu \mathrm{s}$, and obvious fracture began to appear. That is, the adiabatic shear band is transformed into a fracture. It should be noted that the stress drop in the figure at about $5 \mu$ s is caused by the stress wave reflection on the contact surface between the hat specimen and the pressure bar, which leads to the stress unloading.

\section{Concluding remarks}

In this paper, the complex dynamics of spontaneous adiabatic shear band evolution is numerically studied. A thermo-elasticplastic phase-field model has been developed to describe the different stages of ASBs' formation. The Johnson-cook (JC) model including strain hardening, strain rate hardening, and thermal softening is adopted to describe the yield of materials. Damage parameters based on phase-field evolution were introduced and calibrated by experiment results.

The simulation results show that our developed model can well reproduce the formation and evolution of ASBs in typical experiments. As an application, the calibrated model is used to simulate the evolution of the ASB of the hat specimen and the process of its transition to the fracture. The simulation results successfully explain the typical phenomena such as transient "hot spots" and double softening in the experiment.

In conclusion, the model developed in this paper can well predict the initiation, evolution, and interaction of ASBs in metallic materials, and the relevant model parameters can be calibrated by experimental results.

\section{Declaration of Competing Interest}

The authors declare that they have no known competing financial interests or personal relationships that could have appeared to influence the work reported in this paper. 


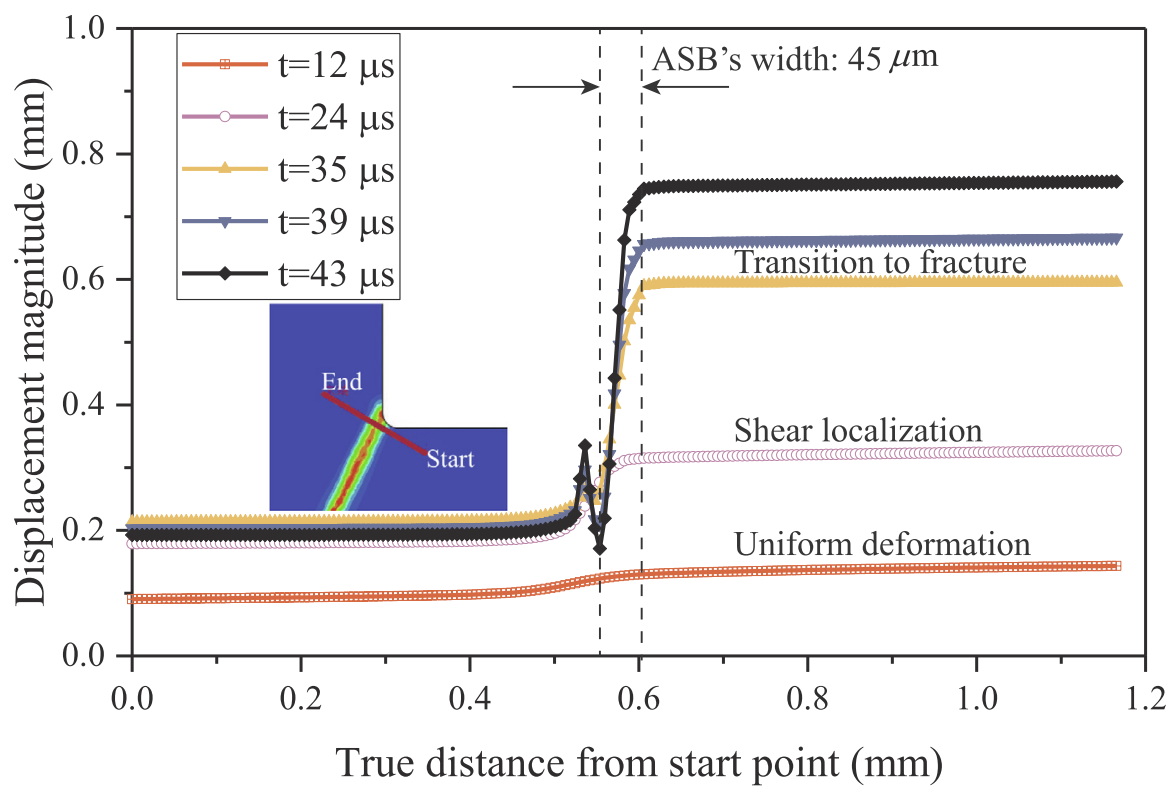

Fig. 10. Displacement magnitude distributions across ASB at different times. As time goes on, the deformation gradually changes from uniform to highly localized.

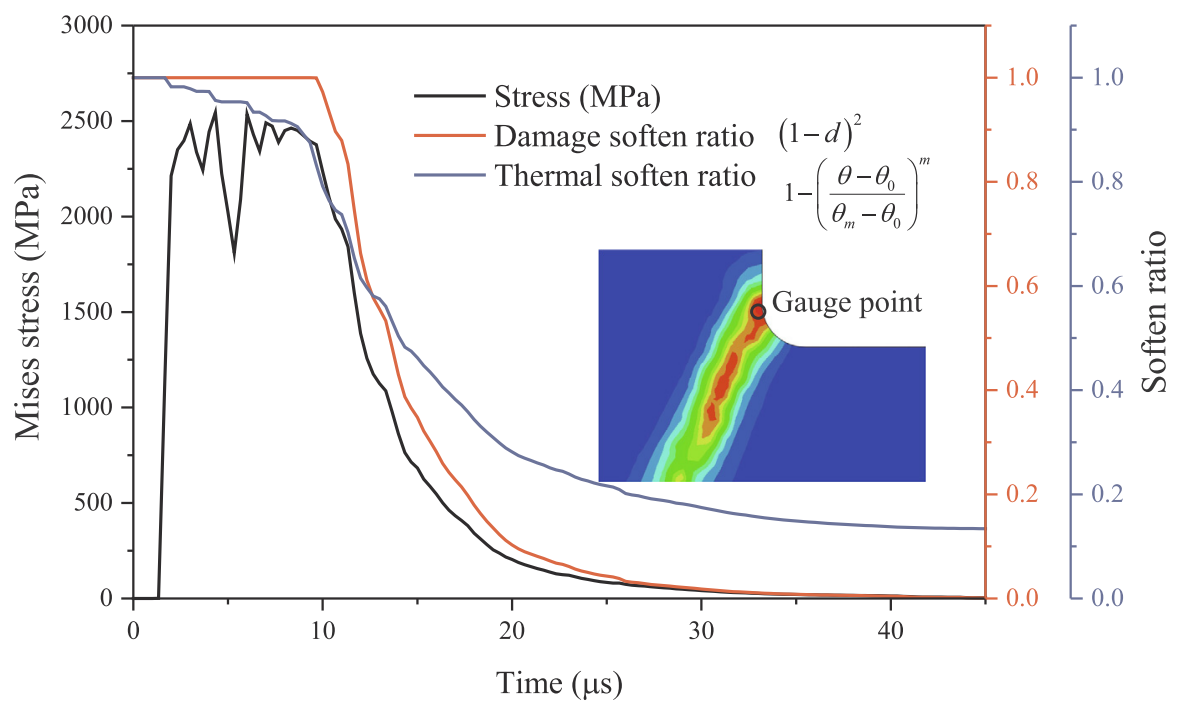

Fig. 11. The curves of stress, thermal softening factor and damage softening factor with time at a gauge point near the interior corner.

\section{Acknowledgment}

This work was supported by Science Challenge Project (No. TZ2018002), the National Natural Science Foundation of China (Grant No. 11532008 and 11972208) and China Postdoctoral Science Foundation (No. 2019M650699).

\section{References}

[1] Zhang Z, Eakins DE, Dunne FPE. On the formation of adiabatic shear bands in textured HCP polycrystals. Int J Plast 2016;79:196-216.

[2] Rittel D. A hybrid experimental-numerical investigation of dynamic shear fracture. Eng Fract Mech 2005;72(1):73-89.

[3] Lieou CKC, Bronkhorst CA. Dynamic recrystallization in adiabatic shear banding: effective-temperature model and comparison to experiments in ultrafinegrained titanium. Int J Plast 2018;111:107-21.

[4] Grady DE, Kipp ME. The growth of unstable thermoplastic shear with application to steady-wave shock compression in solids. J Mech Phys Solids 1987;35(1):95-119.

[5] Wright TW, Ockendon H. A scaling law for the effect of inertia on the formation of adiabatic shear bands. Int J Plast 1996;12(7):927-34.

[6] Molinari A. Collective behavior and spacing of adiabatic shear bands. J Mech Phys Solids 1997;45(9):1551-75.

[7] Marchand A, Duffy J. An experimental study of the formation process of adiabatic shear bands in a structural steel. J Mech Phys Solids 1988;36(3):251-83. 
[8] Zhou M, Rosakis AJ, Ravichandran G. Dynamically propagating shear bands in impact-loaded prenotched plates-i. Experimental investigations of temperature signatures and propagation speed. J Mech Phys Solids 1996;44(6):981-1006.

[9] Guduru PR, Ravichandran G, Rosakis AJ. Observations of transient high temperature vortical microstructures in solids during adiabatic shear banding. Phys Rev E 2001;64(3):036128.

[10] Daridon L, Oussouaddi O, Ahzi S. Influence of the material constitutive models on the adiabatic shear band spacing: MTS, power law and Johnson-Cook models. Int J Solids Struct 2004;41(11):3109-24.

[11] Dorothy HL, Longère P. Modelling of high strain rate adiabatic shear banding induced failure: a comparison of two approaches. Int J Impact Eng 2017;110:219-27.

[12] Liu M, Guo Z, Fan C, Tang T, Wang X, Hu H. Modeling spontaneous shear bands evolution in thick-walled cylinders subjected to external high-strain-rate loading. Int J Solids Struct 2016;97-98:336-54.

[13] Lovinger Z, Rittel D, Rosenberg Z. Modeling spontaneous adiabatic shear band formation in electro-magnetically collapsing thick-walled cylinders. Mech Mater $2018 ; 116: 130-45$.

[14] Wang T, Ye X, Liu Z, Chu D, Zhuang Z. Modeling the dynamic and quasi-static compression-shear failure of brittle materials by explicit phase field method. Comput Mech 2019;64(6):1537-56.

[15] Ziaei-Rad V, Shen Y. Massive parallelization of the phase field formulation for crack propagation with time adaptivity. Comput Methods Appl Mech Eng 2016;312:224-53.

[16] Emdadi A, Fahrenholtz WG, Hilmas GE, Asle Zaeem M. A modified phase-field model for quantitative simulation of crack propagation in single-phase and multiphase materials. Eng Fract Mech 2018;200:339-54.

[17] Zhou S, Zhuang X, Rabczuk T. Phase field modeling of brittle compressive-shear fractures in rock-like materials: a new driving force and a hybrid formulation. Comput Methods Appl Mech Eng 2019;355:729-52.

[18] Miehe C, Hofacker M, Schänzel LM, Aldakheel F. Phase field modeling of fracture in multi-physics problems. part II. Coupled brittle-to-ductile failure criteria and crack propagation in thermo-elastic-plastic solids. Comput Methods Appl Mech Eng 2015;294:486-522.

[19] Chu D, Li X, Liu Z. Study the dynamic crack path in brittle material under thermal shock loading by phase field modeling. Int J Fract 2017;208(1):115-30.

[20] Miehe C, Mauthe S. Phase field modeling of fracture in multi-physics problems. part III. Crack driving forces in hydro-poro-elasticity and hydraulic fracturing of fluid-saturated porous media. Comput Methods Appl Mech Eng 2016;304:619-55.

[21] Duda FP, Ciarbonetti A, Toro S, Huespe AE. A phase-field model for solute-assisted brittle fracture in elastic-plastic solids. Int J Plast 2018;102:16-40.

[22] Geelen RJM, Liu Y, Hu T, Tupek MR, Dolbow JE. A phase-field formulation for dynamic cohesive fracture. Comput Methods Appl Mech Eng 2019;348:680-711. arXiv: 1809.09691.

[23] Wu J-Y, Mandal TK, Nguyen VP. A phase-field regularized cohesive zone model for hydrogen assisted cracking. Comput Methods Appl Mech Eng 2020;358:112614.

[24] Chu D, Li X, Liu Z, Cheng J, Wang T, Li Z, Zhuang Z. A unified phase field damage model for modeling the brittle-ductile dynamic failure mode transition in metals. Eng Fract Mech 2019;212:197-209.

[25] McAuliffe C, Waisman H. A unified model for metal failure capturing shear banding and fracture. Int J Plast 2015;65:131-51.

[26] Arriaga M, Waisman H. Combined stability analysis of phase-field dynamic fracture and shear band localization. Int J Plast 2017;96:81-119.

[27] Feng XQ, Qin QH, Yu SW. Quasi-micromechanical damage model for brittle solids with interacting microcracks. Mech Mater 2004;36(3):261-73.

[28] Feng XQ, Gross D. An approximate scheme for considering effects of microcrack interaction on the overall constitutive relation of brittle solids under complex loading. Acta Mech 2000;139(1):143-59.

[29] Feng XQ, Yu SW. Micromechanical modelling of tensile response of elastic-brittle materials. Int J Solids Struct 1995;32(22):3359-72.

[30] Zeng Q, Tonge AL, Ramesh KT. A multi-mechanism constitutive model for the dynamic failure of quasi-brittle materials. Part I: Amorphization as a failure mode. J Mech Phys Solids 2019;130:370-92.

[31] Zeng Q, Tonge AL, Ramesh KT. A multi-mechanism constitutive model for the dynamic failure of quasi-brittle materials. Part II: integrative model. J Mech Phys Solids 2019;131:20-42.

[32] Malcher L, Mamiya EN. An improved damage evolution law based on continuum damage mechanics and its dependence on both stress triaxiality and the third invariant. Int J Plast 2014;56:232-61.

[33] Xue X, Liao J, Vincze G, Sousa J, Barlat F, Gracio J. Modelling and sensitivity analysis of twist springback in deep drawing of dual-phase steel. Mater Des 2016;90:204-17.

[34] Xu F, Zhang H, Zhao S, Chen C, Cao M, Chen W. Optimized calibration procedure of the damage parameters of 6082-t6 sheets. Materials (Basel) 2018;11(2).

[35] Miehe C, Welschinger F, Hofacker M. Thermodynamically consistent phase-field models of fracture: variational principles and multi-field FE implementations. Int J Numer Meth Eng 2010;83(10):1273-311.

[36] McAuliffe C, Waisman H. A coupled phase field shear band model for ductile-brittle transition in notched plate impacts. Comput Methods Appl Mech Eng 2016;305:173-95.

[37] Amor H, Marigo J-J, Maurini C. Regularized formulation of the variational brittle fracture with unilateral contact: numerical experiments. J Mech Phys Solids 2009;57(8):1209-29.

[38] Wang T, Ye X, Liu Z, Liu X, Chu D, Zhuang Z. A phase-field model of thermo-elastic coupled brittle fracture with explicit time integration. Comput Mech 2020:1-17.

[39] Borden MJ, Verhoosel CV, Scott MA, Hughes TJR, Landis CM. A phase-field description of dynamic brittle fracture. Comput Methods Appl Mech Eng 2012;217-220:77-95.

[40] Borden MJ, Hughes TJR, Landis CM, Anvari A, Lee IJ. A phase-field formulation for fracture in ductile materials: finite deformation balance law derivation, plastic degradation, and stress triaxiality effects. Comput Methods Appl Mech Eng 2016;312:130-66.

[41] Wu J-Y, Cervera M. A novel positive/negative projection in energy norm for the damage modeling of quasi-brittle solids. Int J Solids Struct 2018;139-140:250-69.

[42] Wu J-Y, Nguyen VP, Zhou H, Huang Y. A variationally consistent phase-field anisotropic damage model for fracture. Comput Methods Appl Mech Eng 2020;358:112629.

[43] Wu J-Y. A unified phase-field theory for the mechanics of damage and quasi-brittle failure. J Mech Phys Solids 2017;103:72-99.

[44] Wu J-Y. A geometrically regularized gradient-damage model with energetic equivalence. Comput Methods Appl Mech Eng 2018;328:612-37.

[45] Clayton JD. Dynamic plasticity and fracture in high density polycrystals: constitutive modeling and numerical simulation. J Mech Phys Solids 2005;53(2):261-301.

[46] Bourdin B, Francfort GA, Marigo J-J. The variational approach to fracture. J Elasticity 2008;91(1):5-148.

[47] Zhang Y, Outeiro JC, Mabrouki T. On the selection of Johnson-Cook constitutive model parameters for Ti-6Al-4V using three types of numerical models of orthogonal cutting. Procedia CIRP 2015;31:112-7.

[48] Chen X, Du C. A gradient plasticity model for the simulation of shear localization. Adv Mech Eng 2017;9(9). 1687814017719005.

[49] Teng X, Wierzbicki T, Couque H. On the transition from adiabatic shear banding to fracture. Mech Mater 2007;39(2):107-25.

[50] Couque H. A hydrodynamic hat specimen to investigate pressure and strain rate dependence on adiabatic shear band formation. J Phys IV France 2003;110:423-8. 